\title{
The analysis of Henan hot spring tourism resources development of Lushan Mountain
}

\author{
Li Zhi-Hui, ${ }^{1, a}$ \\ ${ }^{1}$ Department Tourism and hotel management,The Pingdingshan Institute of Education, \\ Pingdingshan Henan 467000, China \\ aLZHCXK@163.COM
}

Key words: Spa Tourism ;The leisure industry;Development Countermeasures

\begin{abstract}
In this paper, now hot spring tourism resources development in Henan Lushan Mountain analysis of existing problems, and puts forward the Countermeasures of developing hot spring tourism resources in line with Lushan Mountain economic development, the results of this study can help Lushan Mountain county government plays a certain reference role in the development of hot spring tourism resources in the.
\end{abstract}

\section{Introduction}

Now, China's hot spring tourism development research mostly geographical scholars, experts and scholars of tourism industry and less attention and Research on hot spring tourism development, so the research achievements in the quantity and quality are not the dominant. Geography scholars studied deeply from Genesis, hot spring tourism resource types, distribution and other aspects, but the hot spring tourism development, industrial development is not much, at present our country existing theory, far behind the pace of development of hot spring tourism industry actual. The author hopes that through this research, can make the development of hot spring tourism can be more tourist attention, eventually forming system, mature theoretical research results, in order to improve the level of theory research on the hot spring tourism industry development of our country, in order to achieve the purpose of theory to guide practice.

\section{Literature Review}

Western countries began to favor of hot spring tourism at the end of the Second World War, spa tourism has gradually become a new force in the tourism industry was strong, tourists began to be hot spring tourism attraction, experts and scholars, corresponding to the western countries the people is becoming more and more fond of hot spring tourism is described, and from the source and process gradually the hot spring tourism development. JohnTowner (1995) the first innovative from another tourism elements of tourist aspect conducts the thorough research to the hot spring tourism development, a more comprehensive summary of the tourist behavior is what aspects of the development and utilization of hot spring tourism plays an important role in [1]. Inskeep (1998) in how to excavate the hot spring tourism destination marketing is not exposed the huge tourist market, mainly in the discipline of marketing for basic research, can be used for development and utilization of hot spring tourism[2].

South China Normal University Urban Planning Research Center (1998) as the main participants issued the "Conghua city overall planning of tourism development of Guangdong Province", the content of hot spring tourism exploitation of Guangdong Province Conghua city to do a comprehensive analysis and summarization, become domestic few research results on the hot spring tourism destination development planning and management of [3]. Wang Yanping, shancunshunci in 2002 based on the results of preliminary studies of our hot spring resources development in-depth discussion, full range of interpretation of the regional characteristics of hot spring tourism resources as the development of capital [4]. 


\section{Analysis of the existing problems in the exploitation of hot spring tourism in Lushan Mountain}

A.Hot spring resources development level is low, existing to the core phenomenon

Through the investigation, found in the existing market in Lushan Mountain tourism products in bath, bathing, and leisure as the main form, but really ignore the spring because of containing metasilicate, fluorine, bromine and other trace elements and health care, health health care medicinal value, access to relevant data shows, Lushan Mountain hot spring water containing trace elemental fluorine was $16.4 \mathrm{mg} / \mathrm{L} \sim 24.85 \mathrm{mg} / \mathrm{L}$, sodium metasilicate content was 86.8mg / L $97.5 \mathrm{mg} / \mathrm{I}$., its content achieved the medical hot mineral water named concentration, while the content of these trace elements and some apparent features of water quality has been far beyond the national drinking water standard, also cannot be used for agricultural irrigation and fish aquaculture, that is to say, says Lushan Mountain hot spring resources with mineral resources property more from some kind of meaning, at the same time, the water temperature can also can be used for energy development and utilization. Therefore, from now on hot spring development level, product level low, development mode innovation of old, hot spring tourism products with local characteristics is very rare, the development of hot spring resources, the low level of formal has been from the core function of a far cry from the hot spring the medicine recuperation from the point of view of value.

\section{B.Through the hot spring tourism market is limited, the market segmentation problems}

At present, the Lushan Mountain hot spring tourist mostly from Pingdingshan city or adjacent city, county. While the source of $60 \%$ come from Pingdingshan city. The number of annual travel to Pingdingshan in only about $6.3 \%$ of hot spring tourism, from this data can be seen in the hot springs tourism increased investment in the construction of the rapid development at the same time and no good possession of the tourism industry in Pingdingshan integrated market share. But the hot spring tourism products of consumer groups to young, female dominated, while the low age class, age class and other market segments are inadequate.

\section{C.The development of spa tourism marketing is poor, management needs to be strengthened}

At present, the Lushan Mountain hot spring tourism marketing has the following disadvantages: first, some hot spring tourism management main body not to study on the tourist market of scientific and rational, subdivision market, blind to sell their products, the result is not satisfactory; second, the market is still not enough to distinguish clearly, the limitations of the tourist market is still strong; third, ways of product sales promotion of the single, a lot of management main body is its own alone, self-produced self marketing, just use their limited marketing channels to sell products, the efficiency of the ground floor, the effect is not obvious.

\section{D.Hot spring scenic spot and serious environmental pollution, environmental protection work is} imminent

Thermal pollution problem: due to medical and bath water temperature at 25 degrees to 30 degrees, while the Lushan Mountain springs exposed temperature was 60 degrees, therefore, must take the hot spring water natural cooling or mechanical cooling to be used, and these emissions into the atmosphere in the heat can not be effectively use, and atmospheric pollution, thermal pollution. Underground hot water because of its utilization degree is low, still having the high temperature tail water discharge, one of which is the thermal pollution source.

Tail water discharge pollution: according to Lushan Mountain hot spring geothermal fluids harmful emission concentration evaluation, according to the standard (GBJ4) stain compounds harmful substances exceed the standard of fluoride in 10.8 to 16.9 times, this is a serious exceed the standard, can not be directly discharged into the local environment. Otherwise it will seriously pollute the water. But in the exploitation and utilization of geothermal areas, many enterprises have not addressed is wanton into scenic spots around on medical, after the bath water, caused certain pollution to the local environment. 


\section{E.Under the Tang overexploitation serious problem}

According to the survey, in the region under the Tang Zhen Limited has been mined 16 geothermal wells, hot spring water resources mined are free supply of 4 hot spring hotels, resorts and local water supply. The exploitation of geothermal resources overload has caused the decline of water level, flow phenomenon disappeared, even in short supply water season. Although the Lushan Mountain county government carried out the rectification in $2010 \mathrm{July}$, the water unit disorder, excessive exploitation of geothermal water phenomenon still exists.

\section{Strategies of developing hot spring tourism in Lushan Mountain of Henan}

\section{A.The introduction of the hot spring tourism talents}

Lushan Mountain County due to long-term economic backwardness, now also belong to the national poverty county, the reason to keep one of the reasons for talents is also important. Many are now engaged in tourism reception service personnel and even the development of hot spring tourism subject and operator are local farmers, while farmers understanding of local resources is deep, but the expression of landscape can not accurately deep cultural connotation, such as the lack of cultural literacy caused by inappropriate tourism development of hot spring tourism resources and error use will hinder the healthy, rapid development of hot spring tourism.

So, it is necessary to introduce outstanding hot spring tourism talent, have the hot spring tourism development of talent Lushan Mountain was intrinsic motivation. Through the high paying job in hot spring tourism management professional college students, doctoral students, through their concept and model of planning innovation for the Lushan Mountain tourism development [5]; also through to the domestic and international hot spring tourism developed area of scriptures, and hire to come to Lushan Mountain exhibit together professionals, make full use of their rich experience the development of hot spring tourism resources to make contributions to the development of spa tourism in Lushan Mountain.

\section{B.Take the road of the development of regional cooperation}

Spa tourism industry can not be alone to complete the task of historical development. Itself is to eat, live, travel, swim, buy, Yu six big industry development. Now our country strongly advocated the development of integration of tourism industry and cultural industry[6], it is to promote tourism in other industries to fully combined, the diversified development of Lushan Mountain hot spring tourism due to traffic underdevelopment and backward economic constraints, the development of tourism is late, and the hot springs tourism because of its large investment, equipment and facilities requirements higher factor developed later, so, at present the country's competitiveness is very limited, so hot spring tourism must and other scenic area joint development, divided two stage:

The first stage is the development of hot spring tourism cooperation Lushan Mountain scenic area and Pingdingshan city adjacent cities and counties. Although Pingdingshan is coal industry city, now is also facing economic transition period, the government has seen the tremendous potential of tourism development, consistent dedicated to the tourism industry as the pillar industry of economic development in Pingdingshan, now the development of Pingdingshan's tourism industry has achieved initial success [7]. With other counties area cooperation can have the following ways: first, in cooperation with the Ruzhou regional development, share the same market and splendid porcelain culture, because both are cultural tourism. Second, to add to the hot spring tourism of Lushan Mountain historical and cultural connotation, can Yexian County to cooperate with the historical development, the shuttle in the Ming Dynasty, Yexian County county people understand the root culture finally after grade bright gentle hot spring culture, have intention to not to effect. Third, can use the combined advantages for development and the Baofeng region adjacent to Baofeng, "the MaJieShuHui", magic, acrobatics and other tourism projects is famous at home and abroad, coupled with Lushan Mountain rich hot spring tourism projects, can the tourism circuit design of rich contents, various forms of.

The second stage, the development of hot spring tourism, Lushan Mountain county and adjacent 
province. Our province is currently the main push "Zheng bian Luo" tourist routes, has a long history, rich connotation of the Song Dynasty, Tang culture make people once entered the picture, a day of millennium, spring from the song Tang across the back for visitors from the dust of history, to return to the modern, as is a kind of tourism product combination form wonderful. Moreover, Zhengzhou, Luoyang and Kaifeng both in the distance of 200 kilometers, convenient transportation, combined with the regional tourism can not only share the tourist, also can enrich "Zheng -bian--Luo" boutique line content.

\section{Reference}

[1]Hirak Behari Routhetal.Balneology, Mineral Water, and Spas in HistoriealPersPeetive[J], Clinies in Dermatology,1996, 14:77-81.

[2]InskeeP.RaPidOxidationofArseniteinaHotSPringEeosystem--YellowstoneNationalPark[J 」.Envi ron.Sei.Teehnol.2001(35):3302-3309.

[3] Weng Liling. Research and development of Chinese and foreign hot spring tourism in comparison to the [J]. market modernization, 2008,12.

[4] Wang Yanping [D]. research Chinese spa area Japan Chiba University degree application essay, 2000:21.

[5] Zou Liang. [J]. study of Dalian City hot spring tourism development problems and Countermeasures of science technology and industry, 200910.

[6] Wang Yuanling. The present situation, problems and Countermeasures of the Northeast Normal University [D]. research, development of hot spring tourism in Jilin Province on 2010.

[7] Fang Youjun,Zhou Min,Cheng Yushen. fuzzy cluster analysis of Tourism Resource based on Object evaluation in hangzhou[J]. Bulletin of Science and Technology. 2008; 24(2): 283-288.. 